\title{
Novel High-Speed Induction Motor for a Commercial Centrifugal Compressor
}

\author{
Wen L. Soong, Member, IEEE, Gerald B. Kliman, Fellow, IEEE, Roger N. Johnson, Raymond A. White, and \\ Joseph E. Miller
}

\begin{abstract}
This paper describes the electrical, mechanical, and metallurgical design, construction, and testing of a novel low-cost high-speed high-efficiency induction motor to drive a new type of small centrifugal compressor in industrial cooling applications. The 28 -shaft-hp $50-\mathrm{kr} / \mathrm{min}$ motor features a unique laminated rotor with a multifunction high-strength copper-alloy cage brazed with a novel process. Relatively thin high-silicon steel laminations were used to achieve low losses and high mechanical strength at low cost. Different heat treatments for the stator and rotor laminations were used to optimize the mechanical and magnetic properties. The preprototype motors achieved about 94\% (electromagnetic) efficiency at the rated point, including inverter harmonics, while meeting cost (less than one-tenth of aerospace practice) and produceability goals.
\end{abstract}

Index Terms-Centrifugal compressor, design, high speed, induction machine.

\section{INTRODUCTION}

$\mathbf{M}$ OST compressors for domestic, commercial, and light industrial air conditioning, refrigeration, or heat pump applications are of the positive displacement type. That is, a volume of refrigerant is captured, mechanically compressed, and ejected into the chiller system. The most common form of positive displacement compressor, particularly for domestic applications, is based on a reciprocating piston. Especially at the lower power ratings, a portion of the piston compressors have been replaced by (positive displacement) rotary compressor units. Screw compressors have also been used for the higher power commercial applications. All of these positive displacement compressor approaches are characterized by pulsating flow and sliding mechanical seals of one form or another. The result is inherent inefficiencies and speeds limited to about $10000 \mathrm{r} / \mathrm{min}$, leading to large physical size.

Centrifugal compressors are intrinsically simple, smooth in operation, and avoid the need for sliding seals. They are com-

Paper IPCSD 99-89, presented at the 1999 Industry Applications Society Annual Meeting, Phoenix, AZ, October 3-7, and approved for publication in the IEEE TRANSACTIONS ON INDUSTRY APPLICATIONS by the Electric Machines Committee of the IEEE Industry Applications Society. Manuscript submitted for review June 1, 1999 and released for publication January 10, 2000. This work was supported by the NIST/ATP Program under Grant 70ANB5H1119.

W. L. Soong was with Corporate Research and Development, General Electric Company, Schenectady, NY 12309 USA. He is now with the Electrical and Electronic Engineering Department, University of Adelaide, Adelaide SA 5005, Australia (e-mail: wlsoong@eleceng.adelaide.edu.au).

G. B. Kliman, R. N. Johnson, and R. A. White are with Corporate Research and Development, General Electric Company, Schenectady, NY 12309 USA (e-mail: kliman@crd.ge.com; johnsor@crd.ge.com; whitera@crd.ge.com).

J. E. Miller, retired, was with GE Industrial Systems, Fort Wayne, IN 46802

USA. He is now at 1214 Windsor Road, Fort Wayne, IN 46825 USA.

Publisher Item Identifier S 0093-9994(00)03175-3.

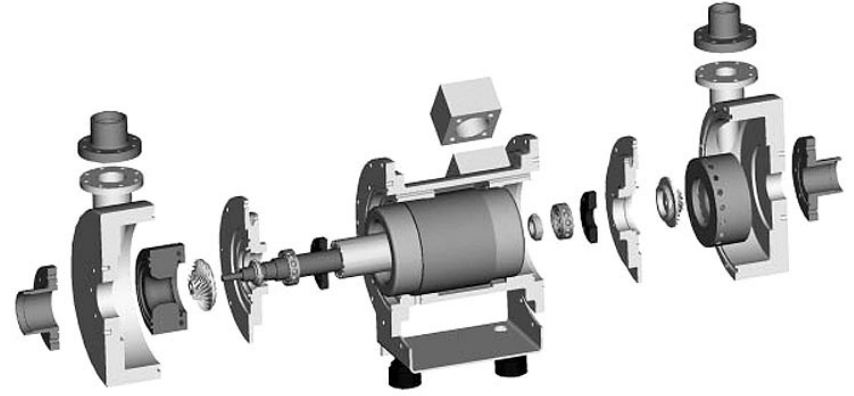

Fig. 1. Exploded view of the high-speed compressor [1].

monly used for high-power applications and have potential for increased efficiency and reduced size in lower power applications, but require very high speeds [1].

A joint effort to develop a small high-speed compressor system was initiated under partial sponsorship of NIST (ATP) and NYSERDA with United Technologies Research (UTRC), General Electric Company (GE), Carrier, Lockheed-Martin, SatCon, Allied Signal, and Dupont participating. This paper describes the GE portion of this effort which was to design, build, and deliver the required high-speed high-efficiency low-cost motor. SatCon independently developed an alternative motor drive to meet the same application requirements based on a permanent-magnet (PM) motor [6].

Brushless dc (PM) motors that could approach the technical performance requirements have, in the past, been built for aerospace applications. In order to achieve a commercially viable system, the challenge was to design a motor that would not only meet the demanding technical and performance requirements but that could be built in a commercial hermetic compressor motor factory with minimal additional tooling and at a selling price that was less than one-tenth that of a comparable aerospace or machine tool spindle drive product. In practical terms, this meant that the motor should cost no more per shaft horsepower than the commercial fixed-speed $60-\mathrm{Hz}$ commodity motors used in current products $(\$ 10-\$ 15 / \mathrm{hp})$.

The two-stage high-speed compressor concept [1] is shown in Fig. 1.

The compressor consists largely of a high-speed high-efficiency motor with two shaft extensions on which are mounted specially designed turbine wheels. The remainder of the structure consists of the mechanical frame, bearing housings, and provisions for cooling the motor, lubricating the bearings, and handling the compressed gases. In this concept, a portion of the refrigerant (in liquid form) is directed through the bearings to 
act as the lubricant (without oil) and then ducted to the outside diameter of the motor yoke where it cools the laminations and becomes mostly gaseous. From there, it passes over one set of stator end windings and into the clearance gap between the stator and the rotor, where it proceeds to the other end of the motor, over the end windings, and out to rejoin the main refrigerant stream. A consequence of this cooling plan is that, in order to minimize pressure drop and windage losses at high speeds, the radial clearance gap between the motor stator and rotor must be relatively large, on the order of $1.27 \mathrm{~mm}(0.050 \mathrm{in})$ on an approximately 5 -cm ( 2 in)-diameter rotor.

The targeted compressor was to have a 25 -ton ${ }^{1}$ rated capacity at $47000 \mathrm{r} / \mathrm{min}$. The resultant requirement on the motor was to deliver $21 \mathrm{~kW}(28 \mathrm{hp}$ ) net power to the (double ended) shaft at an electromagnetic efficiency of $95 \%$. Windage and bearing friction was to be minimized, but in no case could it be more than $0.5 \mathrm{~kW}$. This led to restrictions on the diameter and length of the rotor. The motor had to be capable of sustained operation at $50000 \mathrm{r} / \mathrm{min}$ and full power, but not necessarily with the required efficiency.

For the initial application, a 460-Vrms three-phase supply was assumed as the input to the rectifier/inverter that was to drive the motor. The design of the inverter is not treated here, but was subject to tradeoff studies similar to those done for the motor. The result was a hard-switched insulated-gate-bipolar-transistor (IGBT)-based voltage-source inverter that used pulsewidth modulation (PWM) at about 15 $\mathrm{kHz}$ to deliver almost sinusoidal current waveforms with an approximately constant volts-per-hertz characteristic up to full speed.

\section{TRADEOFF STUDY}

The first task, after definition of the requirements, was to select the proper motor technology. A tradeoff study comparing PM ("brushless dc"), induction, and switched reluctance motors (SRM's) was performed. Each motor design was approximately optimized, within the constraints of the specification, where possible. Fig. 2 shows the cross sections of the preliminary designs of the three types of motors.

The key results of the tradeoff study are summarized in Table I. The same stator outside diameter of $127 \mathrm{~mm}$ (5 in) was assumed for each. The stack height was not restricted, but the first critical speed had to be no lower than $57000 \mathrm{r} / \mathrm{min}$ for mechanical stability and safety. This requirement imposed conditions on the shaft diameter as well.

The PM motor was thought to be the obvious choice, since, without rotor losses, it would have the highest efficiency by far. Also, since the rotor magnetic circuit did not need to be laminated, it could have the stiffest shaft and, hence, the highest critical speed.

A major challenge in PM motors is how to retain the magnets at high speed. The usual method is to use high-energy sintered magnets held against the shaft by a shrunk-on metallic shell or a fiber wrap [6]. However, fiber wrap was ruled out by factory

\footnotetext{
${ }^{1} \mathrm{~A}$ ton is a unit of refrigeration capacity defined as $12000 \mathrm{BTU} / \mathrm{h}(3517 \mathrm{~W})$, but it originally referred to the amount of water that could be turned into ice in a day.
}

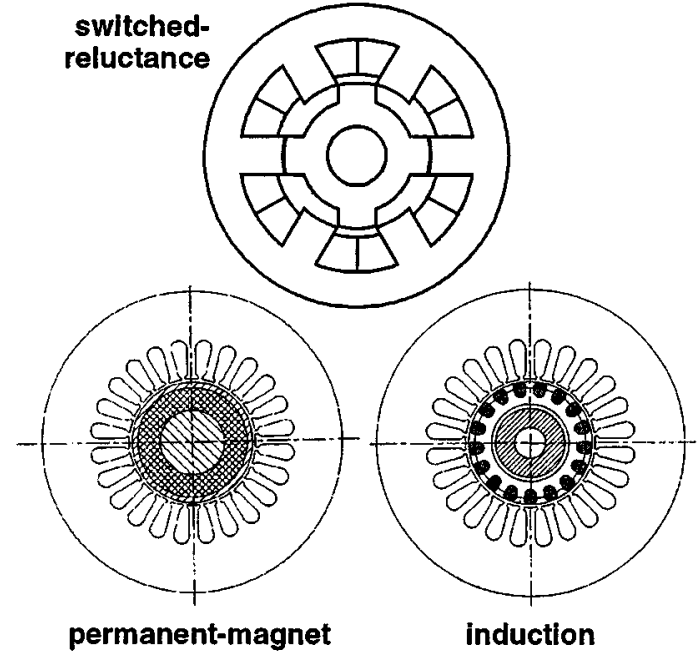

Fig. 2. Preliminary designs of the three motor types used in the tradeoff study.

TABLE I

SUMMARY OF TRADEOFF STUDY RESULTS

\begin{tabular}{l|c|c|c}
\hline Parameter & PM & IM & SR \\
\hline Critical Speed & $80 \mathrm{krpm}$ & $57 \mathrm{krpm}$ & $57 \mathrm{krpm}$ \\
\hline Efficiency & $95+\%$ & $94 \%$ & $93.5 \%$ \\
\hline Relative Cost & 2 & 1 & 1.4 \\
\hline
\end{tabular}

requirements. In the electromagnetic design, it was found that bonded magnets would yield the required performance at considerably lower cost than sintered magnets, but could not withstand the temperatures and stresses of the shell installation. A unique "floating magnet" design [2] was developed to overcome these problems.

In the course of the design study, it was also found that the expected high efficiencies of the PM motor would be considerably reduced by losses in the shell and in the magnets themselves due to stator slotting and inverter PWM effects. Partial fixes for these effects were studied and proposed [2]. In either assembly method, expensive materials and techniques such as inconel, neodymium-iron-boron magnets, and electron-beam welding would be required.

The SRM is always a popular option for high speed operation due to its very simple and robust rotor construction. Unfortunately a reasonable electromagnetic design could not be achieved without reducing the clearance gap considerably below the required value described earlier. Furthermore, in order to achieve a reasonable efficiency, $0.178 \mathrm{~mm}$ (0.007 in) thick laminations were required in both the stator and the rotor. Other issues included: the possible large windage losses due to the salient pole rotor structure and how to maintain tightness of the rotor laminations to avoid movement at high speeds.

The induction motor was attractive as it was familiar to the product department, potentially lowest in cost and could be readily operated without requiring a rotor position or speed sensor. The latter point was an important consideration as any sensor leads would have to penetrate the hermetic enclosure, the sensor would have to work in the refrigerant environment and the sensor would contribute reliability as well as cost problems. There was a possibility of using sensorless control in 


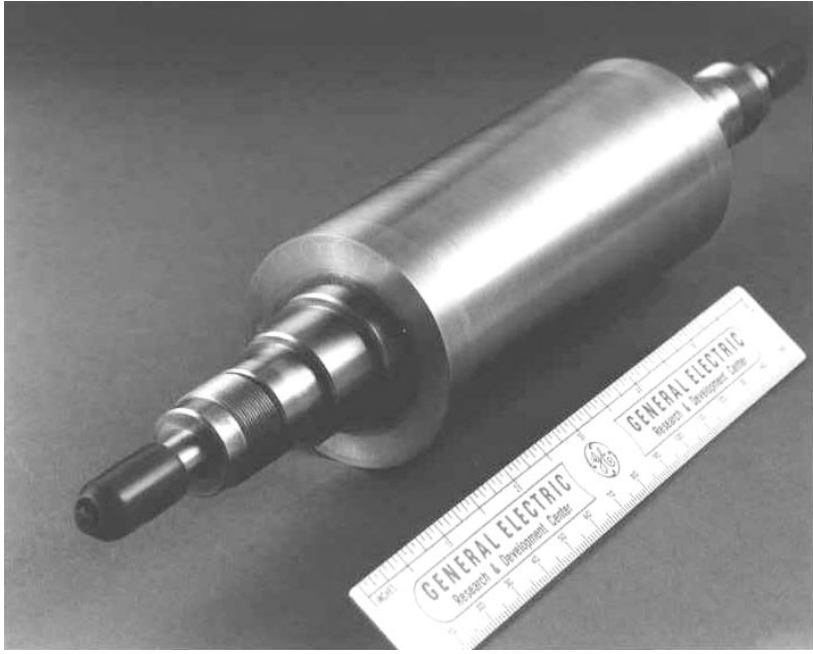

Fig. 3. Photograph of one of the completed preprototype induction motor rotors.

the PM and SRM's but the added complexity and doubts about high speed operation militated against it.

There were also issues with the induction motor, the key one being how to build a reliable and efficient laminated rotor at the required low projected cost.

The major points of the initial trade off study are summarized in Table I. It appeared that the PM motor could just achieve the desired efficiency when using a metallic can magnet restraint. The projected cost was however at least double that of the induction motor and considerably in excess of the maximum allowed. In addition, exotic materials and processes would be required in the factory. The SRM could not meet the requirements and was projected to be about $40 \%$ more expensive than the induction motor, primarily due to the high cost of the very thin laminations.

Based on the results of the trade off study the induction motor was chosen. The solution to the design and manufacturing challenges are the subject of this paper. The projected efficiency did not quite meet the requirement but everything else did. Moreover the projected price was less than that required. The customer (Carrier) determined that price was more important than efficiency and that the efficiency was sufficiently close.

\section{Electrical AND Mechanical Design}

It was essential to achieve a design concept that would be simple and familiar to the motor manufacturing department. In principle, it should also be manufacturable in existing facilities, with perhaps some additional equipment or process outsourcing. The design concept met these constraints and was successfully brought to the demonstration stage (see Fig. 3).

Some of the technical design features (see Fig. 4) required to achieve the goals include the following:

- a laminated rotor as opposed to the solid rotor used in conventional high-speed induction motors; solid rotors are

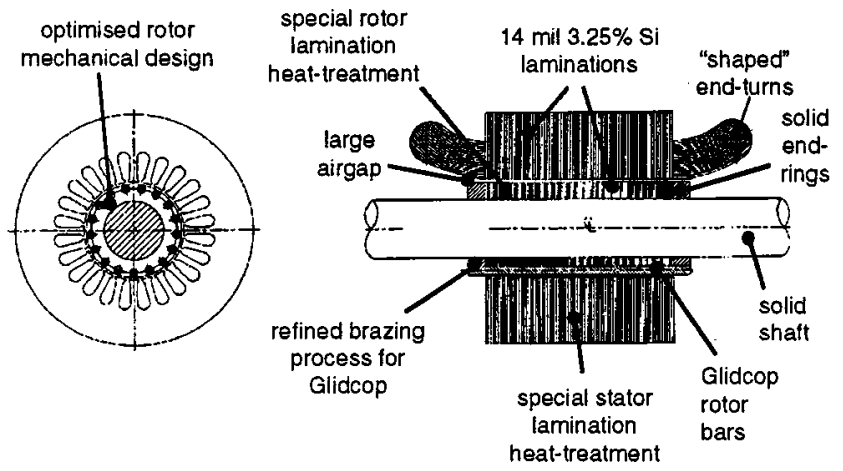

Fig. 4. Key design features of the motor design.

easier to design for high-speed mechanical integrity, but have poorer efficiency;

- high-silicon steel lamination material for low losses and high strength at lowest cost;

- optimization of the lamination heat treatment to tradeoff properties: stator heat treatment for lowest losses, rotor heat treatment for best strength and ductility with adequate electromagnetic properties;

- mechanical finite-element stress analysis for optimum rotor lamination design to minimize stresses during fabrication and high-speed operation;

- advanced high-strength high-conductivity conductor material for the rotor cage and end rings;

- refinement of the low-temperature brazing processes for dispersion-strengthened copper which were optimized and coordinated with the lamination heat treatment;

- minimization of the bearing span by customized compaction and forming of the motor end-winding envelope in order to maximize the rotor critical speed;

- end rings which extend to the shaft to minimize rotor resistance, maximize heat transfer to the shaft, and to provide accurate alignment of the cage components for greater mechanical stability;

- minimization of the stray load losses by reducing the stator slot opening as much as feasible and removing rotor surface smear.

A more detailed discussion of these items follows.

\section{A. Rotor Conductor Selection}

In the rotor design concept, the bars and end rings serve a mechanical as well as an electromagnetic function. The electromagnetic function is the normal one for cage rotor induction motors which is to carry the induced rotor currents. The mechanical function is to preload the rotor stack sufficiently to prevent motion of the laminations during repeated cycling of the compressor. Any movement of the laminations would alter the balance and lead to possible rapid disintegration of the rotor at high speeds. In order to sustain the preload stresses, the bars needed to have about double the yield strength of normal copper. There are several forms of dispersion-strengthened copper. Of these 


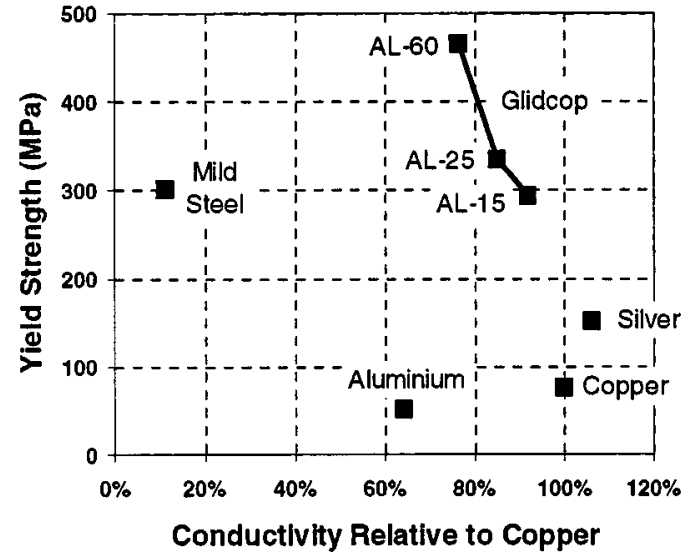

Fig. 5. Tradeoff between conductivity and yield strength for various rotor conductor materials.

forms, Glidcop ${ }^{2}$ offers the highest strength, highest temperature capability, and lowest creep. It also does not exhibit the toxicity of some alloys. This material has been commercially available for more than 20 years.

Fig. 5 shows the tradeoff between conductivity and yield strength for a number of common rotor conductor materials plus three grades of Glidcop (AL-15, AL-25 and AL-60) which differ in the aluminum oxide content.

The AL-15 grade was used for the rotor conductor bars where high conductivity was important. The higher strength AL-60 grade was used for the end rings. This was required due to the possibility of stress concentrations resulting from imperfect joints between the rotor end ring and the bars. The lower conductivity could be compensated for by increased thickness of the end rings. A high-strength backup collar of a material such as stainless steel or metal matrix composite (MMX) is sometimes used to retain the rotor end rings in high-speed induction machines [6]. However, for this application, cost and complexity constraints ruled out the use of a backup collar, hence, the rotor structure had to be self supporting at high speed under all circumstances.

While Glidcop has the required electrical and mechanical properties, it is a difficult material to fabricate. It is made by creating a fine copper powder which is mechanically mixed with aluminum oxide. The mixture is then poured into normal copper tubes, heated, and drawn down to consolidate the material. The normal copper shell must be machined away before finishing to size. Also, due to the dispersed nature of the material, welding by any means is not possible. Any local melting would result in the dispersion being lost, resulting in normal low-strength copper remaining at the joint. The only viable method of joining Glidcop is brazing, which was a problem in itself and is discussed in Section III-C.

\section{B. Stator and Rotor Heat Treatment}

The stator core losses were controlled by using a thin highsilicon lamination material, careful selection of the stator heat treatment, and by limiting the maximum stator flux density in the electromagnetic design.

\footnotetext{
${ }^{2}$ Glidcop is a tradename of SCM Corporation.
}

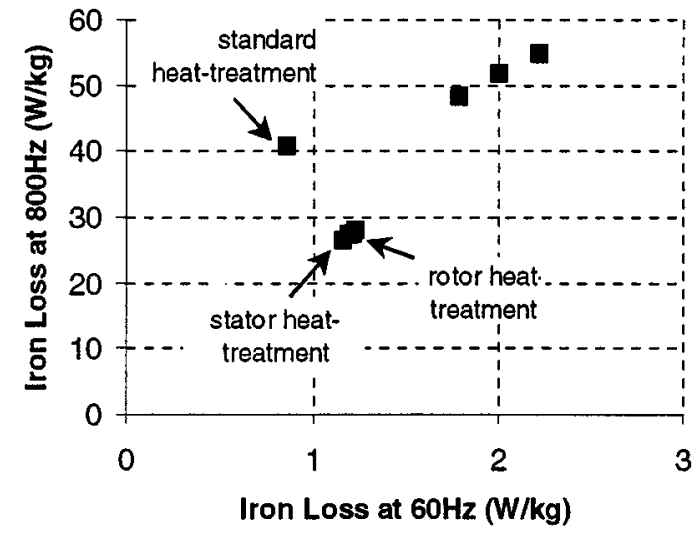

Fig. 6. Tradeoff between the iron losses at $60 \mathrm{~Hz}$ versus the iron loss at 800 $\mathrm{Hz}$ for different heat treatments.

Conventional $60-\mathrm{Hz}$ motors use lamination materials with thicknesses in the range $0.457 \mathrm{~mm}$ (0.018 in) $-0.635 \mathrm{~mm}(0.025$ in). Normally, a motor with a fundamental operating frequency of approximately $800 \mathrm{~Hz}$ would use $0.178-\mathrm{mm}$ (0.007 in) laminations. However, this was ruled out by cost and fabrication capability limitations, and so a compromise of $0.356-\mathrm{mm}(0.014$ in) thickness was used instead.

The high-silicon (3.25\%) non-oriented silicon steel lamination material was received from the supplier in $0.457-\mathrm{mm}(0.018$ in)-thick rolls without an insulating coating. It was then rerolled to the required $0.356-\mathrm{mm}(0.014$ in) thickness. At this point, it had a high yield strength, but was extremely hard and brittle. Both the rotor and stator laminations were oxidized. A very dilute varnish was also applied to the stator laminations to provide a thin insulating coating. For the production version, C5 (inorganic) coated laminations would be used.

To further reduce high-frequency stator core losses, a special heat treatment was developed to produce an optimum grain size in the lamination steel for the nominal operating frequency. Normally, laminations are heat treated to produce large grains in order to minimize hysteresis loss, but at the expected approximately $800-\mathrm{Hz}$ operating frequency, eddy-current losses dominate, so reducing conductivity is beneficial, even though hysteresis losses may increase. A series of batch heat-treatment trials were run in the laboratory to prove the idea. This was later repeated on the factory belt heat-treatment apparatus to fine tune for best results in the production facility. Fig. 6 shows the tradeoff between the iron losses at $60 \mathrm{~Hz}$ versus the iron losses at $800 \mathrm{~Hz}$ for different heat treatments. The iron losses were measured on ring samples using a peak flux density of $0.8 \mathrm{~T}$. The standard heat treatment minimizes the $60-\mathrm{Hz}$ losses. The stator heat treatment was chosen to trade off an increased $60-\mathrm{Hz}$ loss for a reduced $800-\mathrm{Hz}$ loss.

The rotor laminations had a different set of requirements. There was little need for strength in the stator laminations so their heat treatment could be optimized for minimum losses without regard to strength. In the rotor, however, strength (and, also, ductility) is paramount. The iron losses are secondary except very near the surface. Hence, a different heat treatment was developed to achieve the best strength with adequate ductility. It is clear that high yield (and ultimate) strength is needed to 


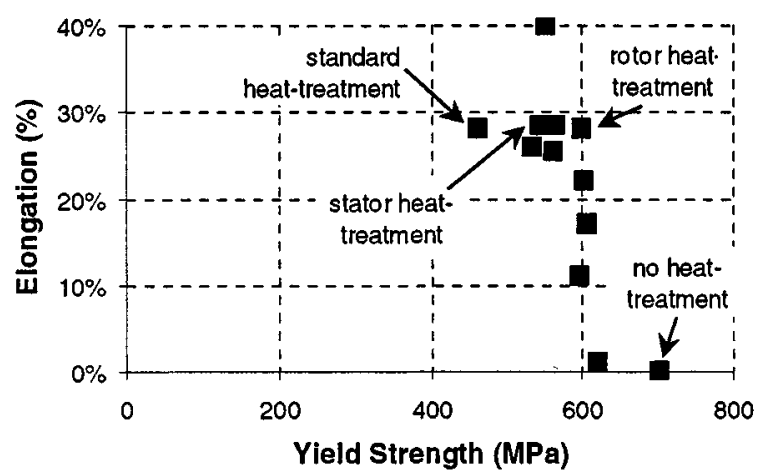

Fig. 7. Tradeoff between yield strength and elongation as the lamination heat treatment was changed.

withstand the high-speed centrifugal forces. A certain amount of elongation or ductility is also needed to enable shrink fits, tolerate some local yielding, and to avoid crack initiation due to scratches and other inevitable mechanical damage. In a manner similar to the stator heat treatment, the rotor heat treatment was proven in the laboratory and refined in the factory.

Fig. 7 shows the tradeoff between yield strength and elongation which was obtained by pull testing samples of lamination materials with different heat treatments. The material is initially very hard (high yield strength) but brittle (low elongation). Heat treatment decreases the yield strength and increases the elongation with the elongation increasing rapidly once the yield strength drops below about $600 \mathrm{MPa}$. The rotor heat treatment was chosen to give the highest yield strength while retaining sufficient elongation.

The laminations for the test motors were cut using a laser cutting system (note that precision punching is planned for the production version). In this phase, the rotor and stator slots and an undersized shaft hole were cut first in the unheat-treated, hard material. The next cut was at the finished stator inside diameter (ID) to produce an oversized rotor. Then, the stator outside diameter (OD) was cut. Heat treatment of the rotor and stator laminations was done separately. This procedure required machining the rotor OD to final dimensions, with some consequent smearing of the rotor surface. It is anticipated that there will be no need for rotor OD machining in the production version.

\section{Brazing}

Due to the dispersed nature of the Glidcop material, it appeared that brazing was the only option. Electron beam welding trials were performed and proved to be unsuccessful.

Brazing is difficult in this application in part because of the following:

- dispersed oxide material makes surfaces hard to wet;

- flux entrapment may cause voids that are stress risers;

- voiding at interfaces due to the silver being extracted;

- embrittlement due to silver migration into the copper;

- a low temperature was required to avoid affecting the heat treatment of the rotor laminations.

To deal with these issues and assure the integrity of the joints, the effects of a number of brazing parameters and preparations were examined on 48 specially prepared test samples. Some samples were of the braze joint alone, but others were mockups of the rotor end region including several laminations. Testing was directed to the following:

- torch versus furnace brazing,

- two levels of clearance between the bars and end ring;

- nickel plating on bars and end rings versus unplated;

- low-temperature versus high-temperature braze alloys;

- two alternative braze alloys for each temperature range;

- flux versus no flux.

Each test sample was "sliced," mounted, polished as a metallurgical specimen, and the integrity of the braze examined. It was found that, for the prototypes, the best combination was using torch brazing, the smaller clearance, no nickel plating, and a low-temperature braze alloy with flux.

In addition to the usual problems of brazing Glidcop discussed above, the brazing alloys and temperatures had to be coordinated with the rotor heat treatment. It was essential that the brazing process did not further heat treat the laminations at the end of the rotor stack, as this would weaken them. This complicated the selection of brazing alloys, surface treatments, and temperatures. Hence, in addition to the tests of joining two pieces of Glidcop, a series of brazing tests was performed on mockups of the end-ring region including laminations. In lieu of strength testing the laminations after brazing, which did not seem feasible, thermocouples were inserted to track the lamination temperature during the test braze and to ensure it did not exceed reasonable values.

\section{Mechanical Design}

Prior to any consideration of material stresses in the rotor, it was deemed necessary to restrict the design to always run below the first critical speed. This is contrary to many design practices [4] and was done to avoid problems with the interference fit between the rotor stack and the shaft and to assure stability of the laminations. This meant that the shaft had to have sufficiently large diameter to achieve the required stiffness with no help from the laminations. This, in turn, required that the solid shaft carry flux. Since maximum slip frequency would be low (around $10 \mathrm{~Hz}$ ), this was not considered to be a problem.

The rotor laminations were required to retain the Glidcop cage bars against centrifugal forces. Hence, the rotor slots had to be closed (see Fig. 3) which was also needed for low windage losses at the cost of some electromagnetic performance. The rotor bar holes shifted the maximum mechanical stress in the rotor lamination due to the shrink fit to the top and bottom of the rotor bars. An extensive series of finite-element method (FEM) stress calculations (see Fig. 8), were carried out to optimize the shrink fit, bar diameter, bridge thickness, and assembly clearance for the lowest value of peak lamination tensile stress.

As described earlier, pull testing on samples of the rotor material was done to establish the parameters of the heat treatment (see Fig. 7). In addition, pull-test samples were included with each production run of the belt heat treatment to assure that the proper characteristics were being achieved. In the high-speed compressor application with its variable-speed capability, there will be considerably less and gentler cycling than in conventional fixed-speed compressors which are operated in an on/off 


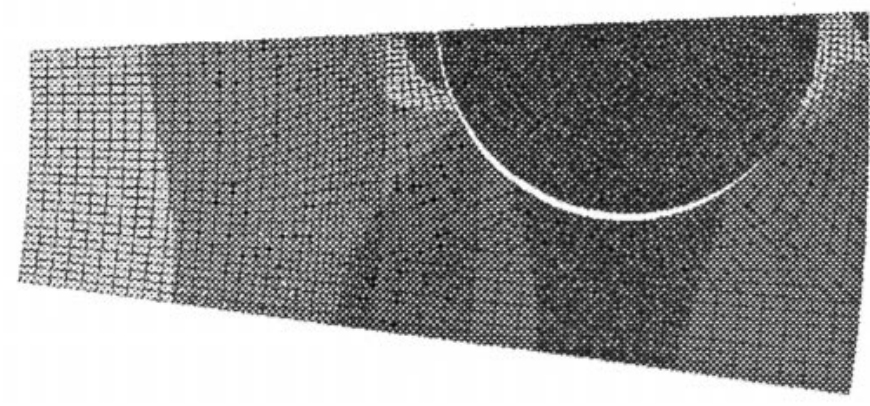

Fig. 8. Typical mechanical finite-element analysis result showing the distribution of rotor lamination stresses.

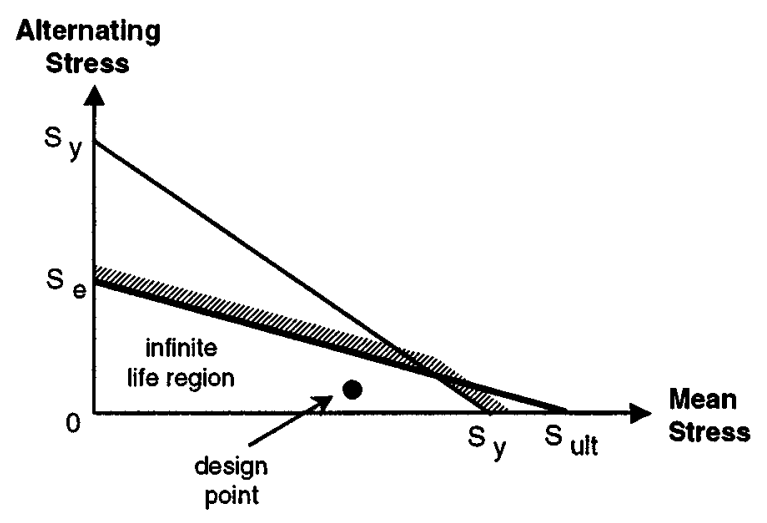

Fig. 9. Goodman (fatigue) diagram for the rotor lamination material.

mode. Nonetheless, a conservative approach was taken to assure that fatigue would not be an issue when pushing so close to the limits of the rotor lamination material. A Goodman diagram (see Fig. 9) for the rotor critical stress areas shows that essentially infinite life is to be expected.

\section{E. Balancing}

Balancing was done to ISO Standard 1940/1 Grade 2.5 in order to keep the maximum unbalance force under $45 \mathrm{~N}$ (10 lbf). Certain areas of the end ring were designated for material removal to enable two-plane balancing on standard balancing machines. Some shifting in balance was noted in spin testing, so a seasoning balance was recommended for production. No shifts in noise or vibration have been observed in the course of extensive dynamometer and chiller testing, which indicate a stable mechanical system.

\section{F. Cooling}

Cooling of the motor is accomplished using the cold refrigerant flow as described in Section I. Computational fluid dynamics (CFD) analyses were carried out by UTRC to estimate the rotor temperatures. Since initial calculations seemed to have little margin, the electromagnetic design was modified for lower torque and, hence, loss density. Thermocouples were distributed in the stator and in the inlet and outlet gas streams for both the dynamometer and chiller tests. They indicated that the stator was well within limits under all conditions. The rotor temperature was estimated from the slip and other performance measurements. The indications are that the rotor temperature was
TABLE II

PRINCIPAL MOTOR DESIGN PARAMETERS

\begin{tabular}{l|l}
\hline Parameter & Value \\
\hline Stator outside diameter & $127 \mathrm{~mm}\left(5^{\prime \prime}\right)$ \\
\hline Rotor outside diameter & $51 \mathrm{~mm}\left(2^{\prime \prime}\right)$ \\
\hline Shaft diameter & $30 \mathrm{~mm}\left(1.183^{\prime \prime}\right)$ \\
\hline Radial airgap length & $1.27 \mathrm{~mm}\left(0.050^{\prime \prime}\right)$ \\
\hline Stack length & $102 \mathrm{~mm}\left(4^{\prime \prime}\right)$ \\
\hline Lamination thickness & $0.36 \mathrm{~mm}\left(0.014^{\prime \prime}\right)$ \\
\hline Winding & Single circuit Y, concentric \\
\hline Stator slots & 24 \\
\hline Rotor slots & 17 (round) \\
\hline Poles & 2 \\
\hline Rated operating voltage & 420 Vll rms \\
\hline Rated operating current & 40 Arms \\
\hline
\end{tabular}

TABLE III

Motor EQuivalent Circuit PARAMETERS

\begin{tabular}{l|c|c}
\hline Parameter & Predicted & Measured \\
\hline $\begin{array}{l}\text { Stator resistance (ohms) } \\
\text { Rotor resistance (ohms) }\end{array}$ & 0.053 & 0.054 \\
\hline $\begin{array}{l}\text { Leakage reactance } \\
\text { (ohms at 60Hz) }\end{array}$ & 0.153 & 0.058 \\
\hline $\begin{array}{l}\text { Magnetizing reactance } \\
\text { (ohms at } 800 \mathrm{~Hz} \text { ) }\end{array}$ & 14.50 & 0.177 \\
\hline
\end{tabular}

lower than expected, so a redesigned motor with shorter stack may be possible.

\section{G. Electromagnetic Design}

The electromagnetic design was carried out using induction motor design software [5] based on the conventional induction motor equivalent circuit. No special corrections were made for the large gap, and only a limited series of manual design optimizations was done. The principal design parameters are given in Table II, and the equivalent circuit parameters as predicted and measured are given in Table III.

Since the compressor was required to operate over a range of loads and speeds, it was important that the efficiency not degrade significantly away from the design point. Fig. 10 shows that this was achieved.

\section{CONSTRuCTION}

A total of six preprototype rotors were fabricated, balanced, and delivered to Carrier Corporation for installation in a special frame for dynamometer testing at UTRC, Hartford, CT, and in a chiller for application testing at Carrier, Syracuse, NY. Detailed design and manufacturing of the stators was carried out at GE Industrial Systems (GEIS), Fort Wayne, IN. Fabrication of the rotor components was done at GEIS and assembly was completed at GE Corporate Research and Development (CRD). Only existing facilities and conventional manufacturing processes were used to fabricate the motors.

\section{TESTING}

Testing was carried out at three locations: spin testing of the rotors was done in a vendor facility nearby CRD, performance 


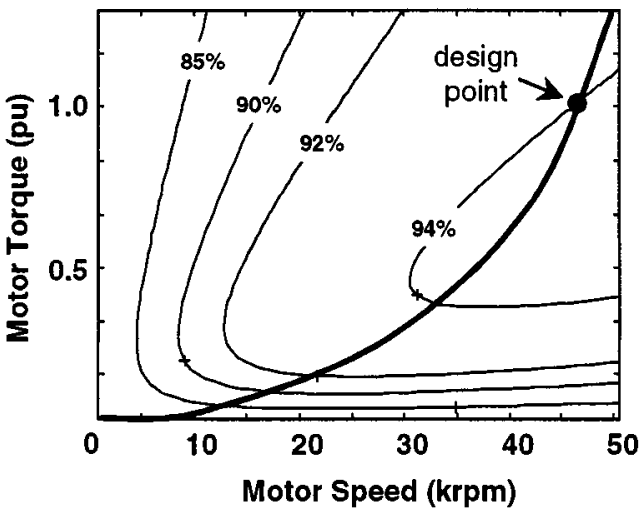

Fig. 10. Estimated electromagnetic efficiency for the induction motor as a function of speed and torque with the expected fan-type compressor load curve.

TABLE IV

CAlculated versus Measured Full-Load Loss Breakdown FOR THE MOTOR

\begin{tabular}{l|c|c}
\hline Parameter & Calculated & Measured \\
\hline Stator core loss & $276 \mathrm{~W}$ & $218 \mathrm{~W}$ \\
\hline Stator copper loss & $309 \mathrm{~W}$ & $313 \mathrm{~W}$ \\
\hline Rotor copper loss & $267 \mathrm{~W}$ & $254 \mathrm{~W}$ \\
\hline Stray load loss & $500 \mathrm{~W}$ & $200 \mathrm{~W}$ \\
\hline Harmonic losses & $210 \mathrm{~W}$ & $264 \mathrm{~W}$ \\
\hline Total electromagnetic losses & $\mathbf{1 5 6 2 ~ W}$ & $\mathbf{1 2 4 9} \mathbf{W}$ \\
\hline Electromagnetic efficiency & $\mathbf{9 3 \%}$ & $\mathbf{9 4 \%}$ \\
\hline
\end{tabular}

testing of the motors using the Lockheed-Martin voltage-source PWM inverter was done at UTRC, and testing of the integrated compressor was done at Carrier.

The following summarizes the test results.

- Three rotors were spin tested above specified maximum speed, with one spun up to $60 \mathrm{kr} / \mathrm{min}$ (20\% overspeed) to verify mechanical integrity and safety. Little distortion was noted.

- No-load and locked-rotor tests were performed to obtain the motor parameters. Good agreement with the design values were achieved for most parameters (see Table III).

- Load tests were performed up to a maximum of two-thirds of rated speed and half rated load power (this was limited by the stability of the water brake dynamometer used).

- Based on test results, the electromagnetic efficiency (including inverter harmonics) will be about $94 \%$ at the rated speed and torque.

- The motors were installed in integrated compressors for further performance and reliability testing at the customer site [1]. Initial results confirm the electromagnetic and mechanical stability at all speeds and loads, as well as efficiency and temperature predictions.

- The full-load loss breakdown as estimated from the dynamometer testing is shown in Table IV. Core losses were a little lower than predicted since the resistivity enhancement produced by the special stator heat treatment was not factored into the software. The largest uncertainty was the stray load losses. At the outset, it was feared that these losses would be about double the value measured. That, and pessimistic assumptions about the effectiveness of rotor cooling, led to a somewhat oversized machine.

\section{CONCLUSIONS}

A preprototype induction motor design was fabricated and demonstrated. It successfully met the cost and efficiency targets for the small, commercial high-speed centrifugal compressor.

The next step is to reoptimize the motor design based on the test results and develop the manufacturing processes used in the motor construction. Other areas of application for a low-cost high-speed motor design, such as machine tool spindle drives, will also be explored [2], [3].

\section{ACKNOWLEDGMENT}

This project was supported by NIST/ATP Program Grant 70ANB5H1119, which was proposed in the initial stages by $\mathrm{T}$. M. Jahns.

The authors wish to thank and acknowledge the contributions of everyone who participated in the design, construction, and testing of this unique motor, including: T. M. Jahns (WEMPEC), J. Hughes (CRD), H. DeLorenzi (QUEST), C. Stephens (CRD), M. Stanley (CRD), Workshops (CRD \& IS), T. Von Holle (ARMCO), G. Huppi (ARMCO), A. Hicks (Arnold), F. Biancardi (UTRC), R. Asserabowski (UTRC), R. Ng (UTRC), D. Pandy (UTRC), D. Brondum (Carrier), J. Materne (Carrier), J. Brown (Lockheed), Schenk-Trebel Corporation, Turbo Technology Services Corporation, T. Miller (SPEED), SCM Corporation, and D. Burchill (Consultant).

\section{REFERENCES}

[1] D. C. Brondum, J. E. Materne, F. R. Biancardi, and D. R. Pandy, "Highspeed, direct-drive centrifugal compressors for commercial HVAC systems," presented at the 1998 Int. Compressor Conf., Purdue Univ., West Lafayette, IN, July 14-17, 1998.

[2] R. N. Johnson, G. B. Kliman, Y. F. Liao, and W. L. Soong, "Rotors with retaining cylinders and reduced harmonic field effect losses," U.S. Patent 5801 470, Sept. 1, 1998.

[3] A. Boglietti, P. Ferraris, M. Lazzari, and F. Profumo, "About the design of very high frequency induction motors for spindle applications," in Conf. Rec. IEEE-IAS Annu. Meeting, 1992, pp. 25-32.

[4] K. Reichert and G. Pasquarella, "High speed electric machines, status, trends, and problems," in IEEE/KTH Stockholm Tech. Conf., Stockholm, Sweden, June 1995, pp. 41-49.

[5] T. J. E. Miller, PC-IMD User Manual. Glasgow, U.K.: SPEED Lab., Univ. Glasgow, 1995

[6] M. Mekhiche, J. L. Kirtley, M. Tolikas, E. Ognibene, J. Kiley, E. Holmansky, and F. Nimblett, "High speed motor drive development for industrial applications," in Int. Electric Machines Drives Conf., Seattle, WA, May 1999, pp. 244-248.

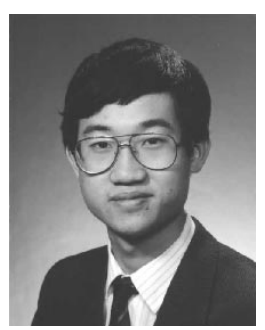

Wen L. Soong (''89-M'90) was born in Kuala Lumpur, Malaysia. He received the B.Eng. degree from the University of Adelaide, Adelaide, Australia, and the Ph.D. degree from the University of Glasgow, Glasgow, U.K., in 1989 and 1993, respectively.

For four years, he was an Electrical Engineer in the Power Controls Program, Corporate Research and Development, General Electric Company, Schenectady, NY, before taking up a teaching position in the Electrical and Electronic Engineering Department, University of Adelaide. His present research interests include permanent-magnet and reluctance motor design and modeling, magnetic bearings, and micromachines. He holds four U.S. patents. 


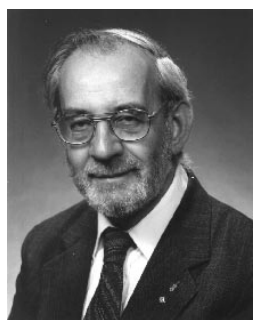

Gerald B. Kliman (S'52-M'55-SM'76-F'92) received the S.B., S.M., and Sc.D. degrees from Massachusetts Institute of Technology, Cambridge, in 1955,1959 , and 1965 , respectively.

Following graduation, he was an Assistant Professor of Electrical Engineering at Rensselaer Polytechnic Institute, Troy, NY. He then had several assignments in the Transportation Systems Division and Nuclear Energy Division of General Electric Company, where he worked on adjustable-speed drives, high-speed linear induction motors, and large electromagnetic pumps. He is currently with Corporate Research and Development, General Electric Company, Schenectady, NY, where he conducts fundamental studies of linear, synchronous, permanent-magnet, and induction motors, advanced drive systems for traction, the development of high-efficiency and high-speed motors, and the application of new and developing magnetic and nonmagnetic materials and insulation. A major emphasis has been the development of fault and incipient fault detection techniques for electric motors and drives. He is an Associate Editor of Electric Machines and Power Systems. $\mathrm{He}$ is the holder of $45 \mathrm{U}$.S. patents and the author of numerous publications.

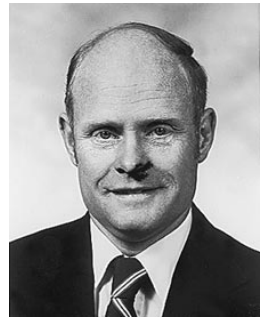

Roger N. Johnson received the B.M.E. degree from Clarkson University, Potsdam, NY, and the M.S.M.E. degree from Rensselaer Polytechnic Institute, Troy, NY, in 1963 and 1965, respectively.

He was a Mechanical Design Engineer with Knolls Atomic Power Laboratory from 1963 to 1978. Since 1978, he has been a Mechanical Design Engineer with Corporate Research and Development, General Electric Company, Schenectady, NY. $\mathrm{He}$ is the holder of 12 U.S. patents.

Mr. Johnson is a Registered Professional Engineer

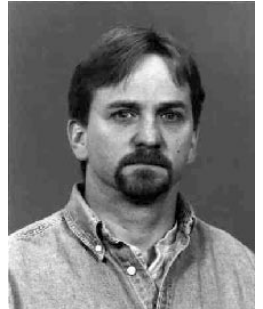

Raymond A. White received the B.S. degree in mechanical engineering from Union College, Schenctady, NY, and the M.Sc. degree in materials engineering from Rensselaer Polytechnic Institute, Troy, NY, in 1984 and 1996, respectively.

Since 1979, he has been involved in materials joining research at General Electric Corporate Research and Development, General Electric Company, Schenectady, NY, where he is currently Senior Professional Staff Engineer responsible for the Joining Laboratory within the Corrosion, Coatings, and Joining Program. He has authored or coauthored 31 General Electric Company internal and open-literature technical papers and publications and is the holder of seven U.S. patents.

Mr. White is a member of the American Welding Society.

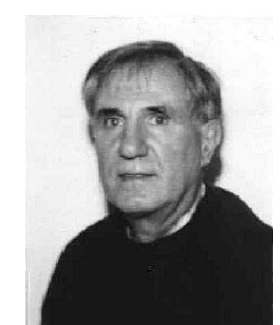

Joseph E. Miller received the B.S. degree from North Manchester College, North Manchester, IN, in 1961.

He was with General Electric Company from 1961 to 1998 , holding individual and managerial positions in engineering and manufacturing. $\mathrm{He}$ transferred to A. O. Smith in 1998 with the sale of the General Electric hermetic compressor motor business to A. O. Smith. He retired in 1999. 